\title{
Tomada de decisão compartilhada no contexto do paciente adolescente em programa de reabilitação
}

\author{
Shared decision-making in the context of adolescent patient in rehabilitation program
}

Toma de decisiones compartida en el contexto del paciente adolescente en programa de rehabilitación

Isabel Cristina Correia ${ }^{1}$

Kalline Carvalho Gonçalves Eler²

Aline Albuquerque ${ }^{3}$

Cíntia Maria Tanure Bacelar Antunes ${ }^{4}$

\begin{abstract}
Resumo
Objetivo: analisar a aplicação da tomada de decisão compartilhada no contexto do paciente adolescente, com base no referencial dos Direitos Humanos dos Pacientes (DHP). Metodologia: baseia-se em pesquisa teórica, que se fundamenta no marco teórico dos DHP, desenvolvido por Albuquerque e Eler e nos estudos sobre a tomada de decisão compartilhada, de acordo com Sullivan. A aplicação do proposto neste artigo foi exemplificada pelo relato de experiência de enfermeiros em hospital de referência em reabilitação no Distrito Federal. Resultados: os Direitos Humanos dos Pacientes como referencial normativo e teórico, bem como a tomada de decisão compartilhada aplicada ao paciente adolescente, asseguram o direito à participação no programa de reabilitação, impactando positivamente nos resultados dos cuidados. Considerações finais: 0 reconhecimento do adolescente enquanto protagonista do seu cuidado em saúde, além de efetivar as determinações de direitos humanos, produz resultados positivos para 0 tratamento, contribuindo para a adesão, o bem-estar, do paciente, e reduz efeitos adversos e ansiedades.
\end{abstract}

\section{Palavras-chave}

Adolescente. Reabilitação. Tomada de decisão compartilhada.

\footnotetext{
Abstract

Objective: to discuss, based on the Patient Human Rights principles, shared decisionmaking applied to adolescent patients. Methods: it is a theoretical research which application was exemplified by the experience of nurses from a rehabilitation hospital in Federal District, Brazil. Results: Patient Human Rights, as a normative and theoretical principle as well as shared decision-making applied to adolescent patients, ensure the right to participate in the

${ }^{1}$ Mestranda, Cátedra Unesco de Bioética, Universidade de Brasília, Brasília, DF, Brasil; enfermeira, Rede Sarah de Hospitais de Reabilitação, Brasília, DF, Brasil. https://orcid.org/0000-0002-4454-4099. E-mail: isabelcrisenfermeira@gmail.com

2 Doutora em Bioética, Universidade de Brasília, Brasília, DF, Brasil; professora, Faculdade de Direito, Universidade Federal de Juiz de Fora, Governador Valadares, Minas Gerais, Brasil. https://orcid.org/0000-0002-5016-579X. E-mail: kallinecarvalho@hotmail.com

${ }^{3}$ Pós-doutora em Direitos Humanos, Universidade de Essex, Colchester, Reino Unido; professora, Programa de Pósgraduação em Bioética, Universidade de Brasília, Brasília, DF, Brasil. https://orcid.org/0000-0002-5568-0790. E-mail: alineaoliveira@hotmail.com

${ }^{4}$ Mestre em Enfermagem, Universidade de Brasília, Brasília, DF, Brasil; enfermeira, Secretaria de Saúde do DF, Brasília, Distrito Federal, Brasil. https://orcid.org/0000-0001-6699-7681. E-mail: cintiatanure@gmail.com
} 
rehabilitation program, with positive impacts to health care outcomes. Conclusion: the recognition of adolescents as protagonists of their own health care, in addition to complying to human rights determinations, produces positive results, contributing to patient compliance, well-being, and reducing adverse effects and anxieties.

\section{Keywords}

Adolescent. Rehabilitation. Shared decision-making.

\section{Resumen}

Objetivo: discutir la toma de decisiones compartidas en el contexto de pacientes adolescentes, en base al marco de Derechos Humanos de los Pacientes, basado en un programa de rehabilitación. Metodología: se basa en la investigación teórica, basada en el marco teórico de Derechos Humanos de los Pacientes desarrollado por Albuquerque y Eler, e en estudios sobre la toma de decisiones compartidas, según Sullivan. La aplicación de lo que se propuso en este artículo fue ejemplificada por la experiencia de enfermería en un hospital de referencia en rehabilitación en el Distrito Federal. Resultados: los derechos humanos de los pacientes como referencia normativa y teórica, así como la toma de decisiones compartidas aplicadas a pacientes adolescentes, aseguran el derecho a participar en el programa de rehabilitación, impactando positivamente los resultados de la atención. Consideraciones finales: el reconocimiento de los adolescentes como protagonistas de su atención médica, además de hacer determinaciones de derechos humanos, produce resultados positivos para el tratamiento, lo que contribuye al cumplimiento del paciente, el bienestar y reduce los efectos adversos y las ansiedades.

\section{Palabras clave}

Adolescente. Rehabilitación. Toma de decisiones compartida.

\section{Introdução}

O conceito de cuidado centrado no paciente tem recebido ampla atenção na área acadêmica desde meados da década de 1950 e é um componente essencial da qualidade do cuidado à saúde (1). Por sua vez, a tomada de decisão compartilhada (TDC) é um processo pelo qual pacientes e médicos atuam juntos, visando à realização de escolhas em cuidados de saúde. Segundo Sullivan (1) a TDC se fundamenta na construção de acordos entre o paciente e o médico sobre as metas que se almejam nos cuidados, contudo, é importante salientar que os objetivos principais são pessoais e específicos do paciente.

Tradicionalmente, a tomada de decisão na saúde era privativa do profissional de saúde e, ao cotejá-la com a TDC, que vem sendo desenvolvida na atualidade, identifica-se a distinção entre os papéis, tanto do paciente, quanto do profissional (2). A TDC pressupõe um papel ativo do paciente, enquanto protagonista do cuidado, e um papel de facilitador do profissional de saúde $(1,2)$, sendo assim, implica a adoção de uma postura colaborativa, na qual paciente e profissionais de saúde atuam em conjunto para fazer escolhas na área da saúde, compartilhando as evidências científicas disponíveis. 
Quanto à paciente criança e adolescente, segundo Hickey (3), no passado não eram considerados legalmente capazes de tomar decisões médicas e eram vistos como incapazes por causa de sua idade, sendo delegada aos pais ou responsáveis a autoridade para consentir ou recusar o tratamento. A autoridade dos pais foi derivada do Direito Constitucional em relação a questões familiares, regra de direito comum e uma presunção geral de que os pais ou responsáveis agiriam no melhor interesse do seu filho. Porém, a partir da metade do século $X X$, os tribunais reconheceram que crianças e adolescentes menores de 18 anos que demonstram maturidade e competência poderiam ter voz na determinação de seu curso de tratamento médico.

Embora a TDC seja o modelo preferencial sob a ótica dos direitos humanos nos cuidados em saúde dos pacientes adultos, em relação aos adolescentes, tal modelo ainda não é amplamente aplicável $(4,5)$. Tal fato pode ser atribuído à escassez de conteúdos de direitos humanos na formação dos profissionais de saúde, bem como à lacuna na formação do profissional de saúde como promotor dos direitos humanos dos pacientes criança e adolescente (5).

Atualmente, o referencial dos Direitos Humanos dos Pacientes (DHP), enquanto corrente teórica da Bioética Clínica, vem sendo utilizado como ferramenta de análise de questões emergentes dos cuidados em saúde. O referencial dos DHP parte das seguintes premissas: os DHP prima facie devem ser respeitados pelos profissionais de saúde; o paciente é ator principal dos cuidados em saúde; e a linguagem dos DHP é a dos direitos e não das obrigações dos profissionais (2). Com efeito, a inserção do referencial teóriconormativo dos direitos humanos na esfera dos cuidados em saúde é essencial para mudança de paradigmas na atuação dos profissionais de saúde em relação ao adolescente como protagonista da sua saúde e como participante ativo na TDC.

Com o fito de contextualizar a aplicação da TDC nos cuidados em saúde de paciente adolescente, optou-se por relato de experiência ocorrida em um serviço de reabilitação. Essa escolha deu-se em razão da reabilitação envolver a mudança no estilo de vida e readaptações na maneira de realizar as atividades de vida diária, o que implica a adesão do paciente. A falta de adesão do paciente poderá acarretar o insucesso no programa de reabilitação, e, por outro lado, a inclusão do paciente na tomada de decisão promove tanto a sua adesão como uma melhor reabilitação (1).

Partindo dessa ótica, este artigo objetiva analisar a aplicação da TDC no contexto dos cuidados em saúde do paciente adolescente, com base no referencial dos DHP, 
empregando como exemplo o emprego da TDC em um programa de reabilitação de paciente adolescente.

\section{Metodologia}

Trata-se de pesquisa teórica, que se fundamenta no marco teórico dos DHP desenvolvido por Albuquerque (2), pesquisadora precursora no Brasil no desenvolvimento de aportes teóricos sobre a aplicação do referencial dos direitos humanos aos cuidados em saúde, e por Eller (6), pesquisadora que se ocupa de estudos sobre os Direitos Humanos Dos Pacientes crianças e adolescentes no encontro clínico. Quanto à tomada de decisão compartilhada, optou-se pelas investigações levadas a cabo por Sullivan, na obra The patient as agent of health and health care (1), por ser pesquisa inovadora acerca da participação e do empoderamento dos pacientes. Com o objetivo de aprofundar a reflexão sobre a aplicação da TDC do paciente adolescente, inicialmente, analisou-se a literatura escolhida pelas autoras; em seguida, aplicou-se os aportes extraídos na literatura citada ao contexto específico do paciente adolescente; por fim, fez-se uso das reflexões prévias para se aprofundar o exame do objeto do artigo, por meio de relato de experiência de TDC de TDC com paciente em reabilitação na unidade de internação pediátrica da Rede Sarah, hospital de referência em reabilitação no Distrito Federal.

Cabe registrar que esta pesquisa não envolve revisão da literatura ou pesquisa bibliográfica, porquanto se trata de pesquisa teórica, que pressupõe a aplicação de determinado referencial teórico a um objeto de estudo (7).

\section{O adolescente no processo de tomada de decisão sobre seus cuidados em saúde}

A adolescência pode ser definida de diferentes formas. Segundo Eisenstein (8), tratase de um período do desenvolvimento físico, mental, emocional sexual e social e pelos esforços que o indivíduo tem em alcançar objetivos relacionados à cultura da sociedade em que ele vive. Cronologicamente, entende-se adolescência como o período de desenvolvimento situado entre a infância e a idade adulta, delimitado pela Organização Mundial da Saúde como a faixa dos 10 aos 19 anos de idade (9). No Brasil, o Estatuto da Criança e do Adolescente (ECA), Lei no 8.069, de 1990, considera adolescência como a faixa etária de 12 a 18 anos de idade (artigo $2^{\circ}$ ) e, em casos excepcionais e quando dispostos na lei, o estatuto e aplicável até os 21 anos de idade (artigos 121 e 142) (10). 
A adolescência abrange, além da puberdade, os componentes psicológicos e sociais característicos dessa fase da vida. Está sujeita, portanto, às influências sociais e culturais. Quanto ao desenvolvimento, sabe-se que a adolescência é um período difícil, onde o indivíduo se prepara para o exercício pleno de sua autonomia (10).

Segundo Garanito e Zaher-Rutherford (11), a capacidade dos adolescentes, enquanto grupo, para tomar uma decisão é intermediária entre a capacidade de crianças e a capacidade dos adultos, e, partindo desse princípio, o envolvimento no processo de tomada de decisão no que concerne à saúde também deveria ocorrer em nível semelhante. Nessas circunstâncias, os prestadores de cuidados de saúde são geralmente os responsáveis por avaliar a capacidade do paciente adolescente e em que medida e situação ele pode vir a tomar uma decisão sobre sua saúde (11).

No que diz respeito à tomada de decisão, é razoável defini-la como o processo de escolha entre duas ou mais alternativas concorrentes, exigindo uma análise de custo e benefício para cada opção, bem como uma estimativa de suas consequências no curto, médio e longo prazo. Uma vez que os resultados das decisões tomadas são incertos, podese dizer que a tomada de decisão envolve a análise de risco e que esse processo está intimamente relacionado com a capacidade de controlar impulsos (11). Portanto, pode-se considerar que a tomada de decisão é indispensável para a adaptação social do indivíduo, e é particularmente difícil quando há maior necessidade de pesar recompensas e/ou perdas imediatas e futuras (11).

De acordo com Lorda (12), os seguintes requisitos são fundamentais para tomar decisões de forma autônoma:

a) falta de coerção externa que restringe significativamente a liberdade de decidir;

b) informações verdadeiras sobre os elementos envolvidos no processo decisório;

c) reconhecimento ético e jurídico, em nível suficiente de habilidades psicológicas, que permitem realizar o processo mental de deliberação;

d) um grau de experiência de vida adequado que nutre sabedoria e prudência no processo de deliberação;

e) um meio ambiente (familiar, social, econômico, político, cultural, etc.) que permite desenvolver as possibilidades de um sujeito deliberativo de forma ativa e positiva (12).

Diante das habilidades que são necessárias para demonstrar a capacidade decisória, observa-se que essa capacidade não é determinada pela idade cronológica e, por isso, foram propostos modelos de capacidade para tomar decisões em saúde. Dentre esses 
modelos, destacam-se a competência Gillick, do Direito britânico, e a doutrina do menor maduro (13).

No modelo da competência Gillick, as habilidades decisionais dos menores de 16 anos são consideradas sem pressuposições abstratas baseadas na idade e, como Herring (13) esclarece, assegura o direito aos menores de 16 anos de consentirem com tratamentos médicos se apresentarem habilidades decisionais para tanto. Para decidir sobre a capacidade da criança, junto com a análise da habilidade decisional, os profissionais de saúde envolvidos no cuidado irão analisar se a decisão também atende aos seus melhores interesses. Para ser considerado capaz, o adolescente deve compreender problemas médicos relevantes; ter uma visão própria e não apenas reproduzir os pontos de vista de outros; compreender as questões morais e familiares envolvidas, bem como as questões médicas; apresentar uma capacidade estável, ou seja, sua aptidão para compreender os diversos fatores concernentes à tomada decisão não pode ser flutuante (13). Nenhum tribunal pode declarar a incapacidade por considerar a decisão do adolescente incorreta. Em relação aos adolescentes de 16 e 17 anos, não se investiga a competência Gillick, pois presumem-se legalmente capazes para consentir sobre sua própria saúde, salvo se o contrário for demonstrado (13).

A doutrina do menor maduro, denominada pela jurisprudência americana, confere ao menor a possibilidade de ser considerado capaz de tomar suas próprias decisões em saúde uma vez preenchidos requisitos que variam conforme o estado $(14,15)$, posto não ser expressamente reconhecida pela legislação federal e pela Suprema Corte dos Estados Unidos. Assim, a análise das jurisdições estaduais demonstra que o modelo do menor maduro pode ser aplicado em três hipóteses: a) a partir de determinada idade, que pode ser 14, 15, 16, 18 anos ou ter completado o Ensino Médio, independente da avaliação da maturidade; b) o menor de determinada idade, 16 anos, ou que seja maduro e capaz de consentir pode fazê-lo se seus responsáveis não estiverem disponíveis ou não desejarem consentir; c) todos os menores que forem maduros e capazes de consentir, a despeito da idade, podem expressar sua vontade que será vinculante (14).

Nos estados que aceitam a exceção do menor maduro, há ainda outra legislação específica que isenta os pais da responsabilidade financeira dos cuidados médicos prestados sem o seu consentimento e apresentam restrições quanto à recusa de tratamento, ponderando esse tipo de decisão com outros interesses do estado, tais como: a) preservar a vida; b) proteger terceiros; c) prevenir o suicídio; e d) manter a integridade ética da 
profissão médica (13). Em geral, nos casos em que a exceção ao consentimento dos pais foi aplicada, além de ter sido avaliada a capacidade sanitária do menor, o tratamento foi realizado em seu benefício e não de terceiros, ou seja, o objetivo do tratamento foi melhorar o estado de saúde do menor e não satisfazer aos interesses dos pais, como por exemplo, nas situações que, devido à crença religiosa, os pais recusavam a transfusão de sangue. Além disso, os procedimentos não colocavam a vida da criança em risco $(14,15)$.

Observa-se, portanto, que maioria dos estados americanos não incorpora o direito do menor de recusar tratamento médico ou tratamento vital, embora existam exceções como o caso de Benny Agrelo, um adolescente de 15 anos que, depois de ter sido submetido a dois transplantes de fígado, recusou-se a tomar o medicamento experimental antirrejeição. 0 medicamento afetava as atividades normais de Benny devido aos efeitos colaterais, como dores de cabeça e irritabilidade, mas sem o fármaco, seu sistema rejeitaria o novo fígado e ele morreria. Apesar do estado da Flórida não admitir o direito de recusa do menor, Benny foi considerado maduro para decidir e conseguiu retornar para casa sem a medicação, falecendo poucos meses depois (16).

De acordo com Partridge (17), no contexto estadunidense, o movimento para apoiar a tomada de decisão independente pelos adolescentes - fornecendo informações a eles e assegurando seu consentimento à parte de seus pais - é incentivado por aqueles entendimentos legais que sustentam que os menores não-emancipados devem geralmente ser considerados como decisão efetiva. A função dos pais nesse caso entra como um paternalismo colaborativo, a fim de proteger os adolescentes de suas próprias escolhas. A família sempre está presente nessas decisões, ainda que a opinião do adolescente seja preservada e respeitada.

Para além dos modelos de capacidade para tomar decisões em saúde anteriormente mencionados, destaca-se o modelo baseado nos Direitos Humanos, que se assenta no reconhecimento da criança - a pessoa menor de 18 anos de idade - enquanto sujeito de direitos. Esse modelo coloca o adolescente como protagonista dos seus cuidados em saúde, sendo dotado de direitos específicos compatíveis com o reconhecimento da sua vulnerabilidade acrescida e o dever ético-jurídico de protegê-lo (18). Diante disso, sublinhase o referencial dos DHP, que também se aplica aos adolescentes, pois, sendo reconhecidos como sujeitos de direito, titularizam direitos específicos no âmbito dos cuidados em saúde.

\section{Aplicação do referencial dos Direitos Humanos do Paciente ao adolescente}


O modelo biomédico foi historicamente construído em um patamar centrado na cultura paternalista de detenção do poder e da informação, na qual a ação era tomada em benefício do paciente, alheia à sua vontade ou desejos. O profissional de saúde decidia, isoladamente, sob o fundamento de ter a melhor percepção do bem para o enfermo. Atualmente, esse modelo vem sendo reconstruído, a partir do reconhecimento de que o paciente é detentor de direitos, em especial, o direito ao respeito à autonomia sobre o seu processo de saúdedoença, podendo assim, participar ativamente de suas escolhas e do seu tratamento (2).

Segundo Eler e Albuquerque (19), o referencial dos DHP é considerado adequado para a proteção dos pacientes, pois, além de impor ao Estado as responsabilidades de respeitar, proteger e realizar os direitos dos pacientes, parte de uma visão ampliada de serviços de saúde, não se restringindo ao acesso aos bens ou aos serviços de saúde. A ênfase é colocada no cuidado que significa zelar pela autonomia, segurança, privacidade, entre outros bens éticos essenciais para se alcançar melhores resultados em saúde e o incremento do bem-estar do paciente (19). Esclarece-se que esse referencial se dirige, precipuamente, ao Estado, enquanto ator responsável por cumprir os comandos de Direitos Humanos quando ratifica um tratado ou convenção. Assim, o Estado obriga-se perante seus jurisdicionados e a comunidade internacional e tem o dever de introduzir, no seu ordenamento jurídico, as normas de Direitos Humanos por ele ratificadas. Especificamente, no âmbito da saúde, compete ao Estado zelar pelo cumprimento dessas normas na prática cotidiana dos profissionais (19).

Enquanto ramo do Direito Internacional dos Direitos Humanos, os DHP referem-se à aplicação dos Direitos Humanos a todos os indivíduos submetidos a cuidados em saúde e seu arcabouço teórico é conformado pelos princípios da dignidade humana, da autonomia relacional, do cuidado centrado no paciente e da responsabilidade $(2,18)$.

Considerando que este trabalho ressalta o protagonismo do paciente adolescente em seus cuidados, afigura-se de suma importância a adoção do modelo do cuidado centrado, pois, o paciente é concebido como o agente fundamental do processo terapêutico dotado de liberdade para deliberar sobre as opções de tratamento, conforme suas condições físicas e psicológicas, seu contexto e seus desejos. O cuidado centrado no paciente vem sendo desenvolvido por escolas diferentes, desde a década de 1950, nos Estados Unidos e, atualmente, tal modelo, inicialmente restrito à relação médico e paciente, expandiu-se para um enfoque multidisciplinar, incrementando a importância do papel de outros profissionais de saúde (20). 
A centralidade no paciente estabelece que ele é agente imprescindível dos cuidados em saúde e o maior beneficiário desses serviços (21). Por isso, cabe ao profissional de saúde compreender o contexto e as especificidades de cada paciente, de modo a identificar suas preferências e atender suas necessidades, construindo, assim, uma relação de confiança. Busca-se, com o cuidado centrado no paciente, suplantar o paternalismo existente na relação entre o profissional de saúde e o paciente, assegurando que o tratamento em saúde atenda às necessidades sociais, emocionais e físicas do paciente, levando em consideração seus valores e preferências $(18,20)$.

Embora o paciente seja o ator central dos seus cuidados, sua autonomia deve ser concebida a partir de uma perspectiva relacional. Isso significa que a identidade do paciente e sua competência decisional são dinâmicas, na medida em que se entrelaçam e são efetivadas conforme seu contexto relacional (18). De acordo com Albuquerque, a família é um elemento crucial no cuidado centrado no paciente, pois, em muitos casos, faz-se necessário envolvê-la no processo terapêutico. Importante ressaltar que cabe ao paciente delimitar quem é sua família e como se dará a participação dos familiares em seu tratamento $(2,18)$.

A TDC está ganhando importância internacionalmente e as razões para essas mudanças incluem o conhecimento crescente dos pacientes sobre doenças e tratamentos por meio da mídia, um número crescente de opções de tratamento disponíveis e as preferências dos pacientes e médicos por um envolvimento mais ativo dos pacientes. A TDC envolve pelo menos um paciente e um profissional de saúde. Ambas as partes tomam medidas para participar ativamente do processo de tomada de decisão, compartilhar informações e, juntos, chegar a uma decisão de tratamento com responsabilidade compartilhada (22)

Esse entendimento aplica-se aos adolescentes que foram reconhecidos enquanto sujeito de direitos, no cenário internacional, a partir da Convenção sobre os Direitos da Criança (CDC), de 1989 (19). A adoção da CDC pela Assembleia da Organização das Nações Unidas (ONU) foi precedida de longos debates que buscaram conciliar as diferentes visões dos Estados sobre a infância e, ao mesmo tempo, estabelecer um rol amplo de direitos que traduzisse uma perspectiva centrada na criança. Assim, a CDC previu, pela primeira vez no Direito Internacional dos Direitos Humanos, o menor de 18 anos enquanto 
sujeito de direitos, modificando a percepção segundo a qual eles seriam objeto de proteção e propriedade dos seus genitores $(19)^{5}$.

O Comitê sobre os Direitos da Criança desencoraja os Estados a introduzirem, por lei ou na prática, limites de idade que restrinjam o direito do adolescente de ser ouvido em todos os assuntos que a afetam, uma vez que a idade em si não é fator determinante para indicar se a opinião do adolescente será tomada em consideração, pois, como acentua o Comitê, os níveis de compreensão dos adolescentes não estão ligados de maneira uniforme a sua idade biológica. Os estudos reiteram que o adolescente não precisa ter conhecimento exaustivo do problema que o afeta, mas sim uma compreensão sobre o assunto suficiente para formar sua opinião própria (19).

O direito à participação assegura que a inclusão do adolescente na tomada de decisão deve ser: i) amigável, de modo que ele se sinta seguro e confortável para externar suas opiniões; ii) seguro, ao ponto de reduzir ao mínimo o risco dos adolescentes sofrerem violência, exploração ou outras consequências negativas; iii) responsável, no sentido de que os adolescentes têm o direito de receber um retorno acerca da forma com a qual sua participação influenciou no resultado; iv) transparente e informativo; v) voluntário, não podendo o adolescente ser obrigado a expressar sua opinião; vi) relevante, ou seja, as questões a respeito das quais os adolescentes expressam suas opiniões devem ter pertinência autêntica para suas vidas $(17,19)$.

Estudos apontam para a dificuldade que alguns adolescentes encontram para expressar suas preferências e vontades, tornando-se necessário um pouco de conhecimento de psicologia infantil para que a comunicação seja efetiva. Por meio de uma linguagem adequada, deve-se fornecer uma descrição da sua condição de saúde e das opções disponíveis de cuidado, bem como dos benefícios e encargos esperados de determinado tratamento $(6,19,24)$. A compreensão das informações pelo adolescente faz parte do conteúdo do direito à informação, de modo que o profissional de saúde precisa empregar

\footnotetext{
${ }^{5}$ A CDC é considerada o primeiro documento a efetivamente reconhecer a criança como sujeito do Direito Internacional, pois a Declaração dos Direitos da Criança de 1959, embora tenha incorporado a linguagem de direitos, não foi constituída como um instrumento jurídico vinculante, sendo apenas uma resolução da Assembleia Geral das Nações Unidas, de modo que é possível inferir apenas a força moral dessa Declaração. Dessa forma, na Declaração de 1959, a criança apenas começa a ser vista como sujeito de direitos, mas isso, somente será concretizado, de fato, na CDC. Além disso, como explica Van Bueren (23), o aumento dos esforços em direção à elaboração da CDC deu-se, entre outras razões, justamente porque os Estados reconheceram que tanto a Declaração de 1959 quanto a Declaração Universal de Direitos Humanos de 1948 não eram instrumentos jurídicos suficientes para abarcar as peculiaridades em torno dos direitos da criança, sendo o mesmo raciocínio aplicável ao Pacto Internacional Sobre os Direitos Civis e Políticos, que apenas reconheceu os direitos à proteção, ao registro, ao nome e a nacionalidade da criança. Assim, sedimentou-se o entendimento de que seria necessário providenciar um documento de direitos que fosse mais apropriado às demandas especiais das crianças e facilmente acessível por aqueles que atuavam no campo dos direitos da criança. Em suma, era necessário introduzir um documento jurídico vinculante centrado na criança enquanto sujeito de direitos.
} 
esforços para que o adolescente entenda claramente os riscos materiais concernentes a determinado tratamento. A revelação das informações também promove a capacidade do adolescente para tomar decisões sobre cuidados em saúde/ aumenta sua confiança nos profissionais envolvidos em seus cuidados/ e incrementa a qualidade do cuidado $(19,24)$.

A tomada de decisão acerca dos cuidados em saúde corresponde ao nível mais elevado de participação e denota o respeito das capacidades evolutivas dos adolescentes, previsto no art. 5ำ da CDC. A expressão parasse refere aos processos de amadurecimento e de aprendizado por meio dos quais crianças e adolescentes adquirem, progressivamente, conhecimento, competências e compreensão, em particular, a compreensão dos seus direitos e como eles podem ser realizados da melhor forma (25).

O direito do adolescente de exercer níveis cada vez mais crescentes de responsabilidades não anula as obrigações que incumbem aos pais e ao Estado, notadamente, a obrigação de protegê-lo. O abandono gradual da proteção da família, somado à relativa inexperiência e falta de poder dos adolescentes pode ocasionar violações aos seus direitos. Por essa razão, o Comitê dispõe que a busca pelo equilíbrio entre o respeito pelo desenvolvimento das capacidades evolutivas dos adolescentes e os níveis adequados de proteção deve considerar uma série de fatores que influenciam a tomada de decisão, por exemplo, o nível de risco envolvido; a possibilidade de exploração; a compreensão do desenvolvimento do adolescente; a consideração das suas experiências individuais; e o reconhecimento de que as habilidades decisionais diferem conforme o tipo de decisão a ser tomada $(19,25,26)$.

\section{Relato de experiência na TDC com adolescentes no programa de reabilitação}

Segundo Martini (27), podemos definir a reabilitação como um trabalho com aspectos educacionais, sociais e terapêuticos em que uma pessoa que sofre algum tipo de deficiência procura restabelecer ou criar recursos para retomar as atividades de sua vida da melhor forma possível. Consistirá no trabalho lento e contínuo que leva, passo a passo, ao melhor discernimento das próprias limitações e possibilidades.

Sistemas de saúde, com concepção holística e abrangente, estabelecem conceitos medulares ${ }^{6}$ do cuidado centrado no paciente e na família em sua visão de trabalho. A Rede Sarah de Hospitais de Reabilitação implementa uma concepção moderna de reabilitação, trazendo o paciente como ponto central do cuidado em seus princípios (28). Trata-se de uma

\footnotetext{
${ }^{6}$ Utilizados em reabilitação, têm conotação de essencial, ou seja, são conceitos essenciais.
} 
instituição que se iniciou em Brasília, na década de 1960, com método de neurorreabilitação, numa abordagem que incorpora a família e o contexto de cada pessoa no processo de neurodesenvolvimento. A terapia de reabilitação não está restrita apenas ao ambiente hospitalar, é necessário ter como objetivo a transitoriedade de acontecimentos durante o processo de tratamento, onde cada momento do paciente, ao longo do dia, deve ser organizado para estimular seu desenvolvimento.

Tradicionalmente, o enfoque dos programas de reabilitação costumava voltar-se para a avaliação das perdas funcionais decorrentes de acidente ou doença (29). Já o programa de reabilitação segundo o método Sarah atua na potencialização das funções preservadas, ou seja, é determinado pelas capacidades que permaneceram, permitindo o alcance de um objetivo funcional, independentemente da maneira como o indivíduo o realize ou execute (28). Portanto, dá-se a importância ao programa individualizado e personalizado, onde o paciente é o centro do cuidado. A atuação da equipe de reabilitação concentra-se predominantemente no que aquele indivíduo consegue realizar e não naquilo que deixou de fazer. De acordo com Braga e Campos da Paz (30):

MÉTODO SARAH: integra equipe multidisciplinar e família para que, juntos, encontrem caminhos para facilitar a aprendizagem e os processos do neurodesenvolvimento da criança e do adolescente, tomando como base, motivações, capacidades e interesses individuais dentro de seu contexto familiar e sociocultural. (30)

Neuropsicólogos, médicos, enfermeiros, fisioterapeutas, terapeutas ocupacionais, professores, nutricionistas, fonoaudiólogos, demais profissionais buscam constantemente aprimorar o cuidado em saúde para tornar possível se colocar diante da fragilidade do outro sem impor uma relação de poder para que a condição de sujeito possa desenrolar-se ao longo do processo de reabilitação.

Pais, amigos, familiares e pessoas que formam a rede de apoio do paciente adolescente são componentes importantes que poderão auxiliar a equipe de profissionais de saúde na integração do paciente na comunidade no momento da alta hospitalar. Tratase, portanto, da subversão do conceito tradicional de reabilitação, em uma vertente de vanguarda, a reabilitação ecológica (28). Nessa ótica, insere-se a interação do adolescente com a família, do jovem com seu grupo, do idoso com suas áreas de interesse, entre outras.

No programa de reabilitação em avaliação, o adolescente possui metas a alcançar e a tomada de decisão compartilhada é algo essencial na relação entre o paciente e a equipe. A mudança no estilo de vida e readaptações na maneira de realizar as atividades de vida 
diária implicam necessariamente na adesão do paciente em mudar de conduta, pois, caso não haja adesão, o programa de reabilitação não alcançará resultados satisfatórios, haja vista que se trata de uma abordagem personalizada, no qual o adolescente é inserido no processo e na discussão de saúde-doença que o compromete, dentro do modelo centrado no paciente, por meio da tomada de decisão compartilhada. O programa de reabilitação ao adolescente é voltado para o autocuidado e para o esclarecimento da patologia, fornecendo informações adequadas e compatíveis ao seu entendimento. Nessa fase, visa-se preservar o direito à privacidade, posto que se encontra em uma etapa de formação de sua personalidade e independência (características da fase da adolescência), concomitante a mudanças físicas e de imagem, que corroboram para os processos de construção da identidade e da cidadania, os quais costumam ser determinantes na experiência das pessoas e para a sua saúde (31).

Adolescentes com deficiência física no programa de reabilitação da Rede Sarah passam por um processo minucioso de avaliação com uma equipe interdisciplinar, onde é realizada a anamnese detalhada e levantamento dos diagnósticos e metas a serem traçadas, com delimitação do tempo para cada uma delas. Demandas de relevância físicas, motoras, cognitivas e sociais são colocadas como metas, com enfoque no autocuidado. Para que ocorra a TDC, é essencial que todas as informações do processo sejam disponibilizadas ao paciente e à família ou responsável legal cabe o papel de apoiador e mediador do processo de reabilitação. A educação e o lazer, por exemplo, são temas pouco abordados pela equipe, mas estão constantemente nas prioridades do adolescente, o que denota a importância de abranger os valores do paciente na TDC.

A satisfação e a adesão do paciente às orientações - quando é ele um participante ativo nas suas decisões - são resultados adquiridos nessa prática diária.

\section{Considerações finais}

Este estudo demonstrou que, no âmbito do modelo do cuidado de saúde alicerçado na tomada de decisão compartilhada (TDC), o adolescente, embora vulnerável em virtude da sua maturidade em desenvolvimento, também é detentor de direitos, que lhe asseguram um papel central na esfera dos cuidados em saúde. Igualmente, sob o enfoque dos Direitos Humanos do Paciente (DHP), o adolescente é reconhecido como protagonista dos seus cuidados, dotado de direitos específicos, compatíveis com o reconhecimento da sua vulnerabilidade acrescida e o dever ético-jurídico de protegê-lo. $O$ envolvimento do 
adolescente no seu próprio cuidado importa no reconhecimento do seu direito de participar e tomar decisões em relação aos seus próprios cuidados. Nesse contexto, a família não é excluída do processo de tomada de decisão. Por essa razão, as decisões sobre cuidados em saúde não são simplesmente decisões individuais; elas afetam os outros e são eventos interpessoais ou familiares. Neste trabalho, sustenta-se que é possível obter um processo de reabilitação compartilhado e centrado no paciente, promovendo a autonomia do adolescente, proporcionando seu engajamento nos cuidados e a satisfação com o tratamento proposto, por meio do uso da TDC. Nos cuidados de reabilitação, é imprescindível dar voz ao paciente. O reconhecimento do adolescente enquanto protagonista do seu cuidado em saúde, além de ser compatível com as determinações de Direitos Humanos, produz resultados positivos para o tratamento, satisfação e bem-estar, podendo reduzir efeitos adversos e ansiedades.

\section{Referências}

1. Sullivan, Mark D. The Patient as Agent of Health and Health Care [Internet]. Oxford University Press; 2018 [acesso em 1.dez.2019]. doi: https://doi.org/10.1093/med /9780195386585.001.0001

2. Albuquerque A. Direitos Humanos dos Pacientes. Curitiba: Juruá; 2016. ISBN: 9788536255194

3. Hickey K. Minors' Rights in Medical Decision Making. Jonas Health Law Ethics Regul [Internet]. 2007 [acesso em 1.dez.2019]; 9(3):100-4. doi:

https://doi.org/10.1097/01.NHL.0000287968.36429.a9

4. Coyne I, Amory A, Kiernan G, Gibson F. Children's participation in shared decisionmaking: Children, adolescents, parents and healthcare professionals' perspectives and experiences. Eur J Oncol Nurs [Internet]. Elsevier Ltd, 2014 [acesso em 5.jan.2020];18(3):273-80. doi: https://doi.org/10.1016/j.ejon.2014.01.006

5. Coyne I. Children's participation in consultations and decision-making at health service level: a review of the literature. Int J Nurs Stud [Internet]. Elsevier; 2008 Nov [acesso em 5.jan.2020];45(11):1682-1689. doi:https://doi.org/10.1016/j.ijnurstu.2008.05.002

6. Eler K, Albuquerque A. Direitos humanos da paciente criança. Cadernos lberoAmericanos de Direito Sanitário [Internet]. 2019 jan./mar. [acesso em 1.dez.2019]; 8(1): 3652. doi: https://doi.org/10.17566/ciads.v8i1.509

7. Adom D, Adu Agyem J, Emad H. Theoretical and conceptual framework: mandatory ingredients of a quality research. International Journal of Scientific Research [Internet]. Jan. 2018 [acesso em 20.dez.2019]; 7(1):158-172. Disponível em:

https://www.worldwidejournals.com/international-journal-of-scientific-research- 
(IJSR)/article/theoretical-and-conceptual-framework-mandatory-ingredients-of-a-qualityresearch/MTM5NDE=/?is=1

8. Eisenstein E. Adolescência: definições e critérios. Adolesc Saude. 2005;2(2):6-7

9. OMS. Saúde do adolescente [Internet]. Genebra: Organização Mundial da Saúde; 2004 [acesso em 20.dez.2019]. Disponivel em: http://www.adolescencia.org.br/site-ptbr/adolescencia

10. Brasil. Estatuto da Criança e do Adolescente. Lei oํ 8.069/90. Brasília, DF: Congresso Nacional; 1990.

11. Garanito MP, Zaher-Rutherford VL. Adolescent Patients and The Clinical Decision About Their Health. Rev. Paul. Pediatr. [Epub]. 2019 Jun [acesso em 30.dez.2019]; 37(4):503-509. Disponível em: http:www.scielo.br/scielo.php?script=sci_arttext\&pid=S010305822019000400503\&Ing=en. doi: https://dx.doi.org/10.1590/1984-0462/;2019;37;4;00011

12. Lorda PS. Madurez, capacidade y autonomia. Eidon [Internet]. 2014; 41:3-11. doi: https://doi.org/10.13184/eidon.41.2014.3-11

13. Herring J. Medical law. New York: Oxford: University Press; 2011. 307 p.

14. Benston S. Not of Minor Consequence? Medical Decision-Making Autonomy and the Mature Minor Doctrine. Indiana Health Law Rev [Internet]. 2016 [acesso em 1.dez.2019]; 13(1): 1-14. doi: https://doi.org/10.18060/3911.0011

15. Coleman DL, Rosoff PM. The Legal Authority of Mature Minors to Consent to General Medical Treatment abstract. Pediatrics [Internet]. 2013 [acesso em 5.jan.2020]; 131:78693. doi: https://doi.org/10.1542/peds.2012-2470

16. Driggs AE. The mature minor doctrine: do adolescents have the right to die? Health Matrix; 2001. p. 687-717.

17. Partridge B. Adolescent Pediatric Decision-Making: A Critical Reconsideration in the Light of the Data. HEC Forum [Internet]. 2014 [acesso em 20.jan.2020]; 26(4):299-308. doi: https://doi.org/10.1007/s10730-014-9250-8

18. Albuquerque A. Capacidade Jurídica e Direitos Humanos. Rio de Janeiro: Lumen Juris; 2018. 284 p. ISBN-13: 978-855191025

19. Eler $\mathrm{K}$, Albuquerque $A$. Direito à participação da criança nos cuidados em saúde sob a perspectiva dos Direitos Humanos dos Pacientes. Revista Iberoamericana de Bioética [Internet]. 2019 [acesso em 20.jan.2020]; 9:01-15 doi: https://doi.org/10.14422/rib.i09.y2019.001

20. Cohen J, Ezer T. Human Rights in patient care: A theoretical and practical framework. Health Hum Rights. 2013;15(2):7-19. 
21. Pluut B. Differences that matter: developing critical insights into discourses of patientcenteredness. Medicine, Health Care and Philosophy [Internet]. Jun. 2016 [acesso em 1.dez.2019];19(4):501-515. doi: https:// doi.org/10.1007/s11019-016-9712-7

22. Doherr H, Christalle E, Kriston L, Härter M, Scholl I. Use of the 9-item Shared DecisionMaking Questionnaire (SDM-Q-9 and SDM-Q-Doc) in intervention studies-A systematic review. PLoS One [Internet]. 2017 Mar 30 [acesso em 5.jan.2020];12(3):e0173904. doi: https://doi.org/10.1371/journal.pone.0173904

23. Van Bueren $\mathrm{G}$. The international law on the rights of the child. The Hague: Martinus Nijhoff; 1998. 435 p.

24. Hyun I. When adolescents "mismanage" their chronic medical conditions: An ethical exploration. Kennedy Institute of Ethics Journal [Internet]. 2000 [acesso em 5.jan.2020]; 10 (2):147-163. doi: https://doi.org/10.1353/ken.2000.0013

25. Lansdown G. The evolving capacities of the child [Internet]. Unicef; 2005 [acesso em 20.dez.2019]. Disponível em: https://www.unicef-irc.org/publications/pdf/evolving-eng.pdf

26. Eler K. Capacidade sanitária da criança sob a perspectiva dos Direitos Humanos Dos Pacientes [Tese]. Brasília: Universidade de Brasília; 2019. 200 fls.

27. Martini, De A. Reabilitação, ética e técnica. Ciência \& Saúde Coletiva [Internet]. 2011 [acesso em 1.jan.2020]; 16(4), 2263-2269. doi: https://doi.org/10.1590/S141381232011000400025

28. Rede Sarah de Hospitais de Reabilitação. Associação das Pioneiras Sociais. Nossos princípios [Internet]. Brasília, 2019 [acesso em 1.dez.2019]. Disponível em: http://sarah.br/

29. Bampi LNS, Guilhem D, Alves ED. Modelo social: uma nova abordagem do tema da deficiência. Rev. Latino-Am. Enfermagem [Internet]. Agosto de 2010 [acesso em 23.maio.2020]; 18 (4): 816-823. Disponível em:

http://www.scielo.br/scielo.php?script=sci_arttext\&pid=S010411692010000400022\&lng=en. https://doi.org/10.1590/S0104-11692010000400022

30. Braga LW, Campos da Paz JRA. Método Sarah: reabilitação baseada na família e no contexto da criança com lesão cerebral. Ed. Santos; 2008. ISBN 9788572887151

31. Ayres JR de CM et al, editores. Caminhos da Integralidade na atenção primária à Saúde: recursos conceituais e instrumentos práticos para a educação permanente da equipe - Adolescência e Juventude [Internet]. São Paulo: Centro de Saúde Escola Samuel B. Pessoa/FMUSP; 2012 [acesso em 19.jan.2020]. Disponível em: https://www.scielo.br/pdf/icse/v16n40/aop2212.pdf 


\section{Colaboradores}

Correia IC contribuiu com a concepção e desenho do artigo, análise e interpretação dos dados, redação e aprovação da versão final. Eler KCG contribuiu com a redação, revisão crítica e aprovação da versão final do artigo. Albuquerque A contribuiu com a concepção, desenho e revisão crítica do artigo. Antunes CMTB contribuiu com a revisão crítica do artigo.

Como citar este artigo:

Correia IC, Eler KCG, Albuquerque A, Antunes CMTB. Tomada de decisão compartilhada no contexto do paciente adolescente em programa de reabilitação. Cadernos Ibero-Americanos de Direito Sanitário. 2020 out./dez.; 9(4): 171187.

https://doi.org/10.17566/ciads.v9i4.617 